\title{
¿Cómo incrementar la seguridad y la efectividad en DES?
}

\author{
Guido Grajales-Figueroa* \\ Instituto Nacional de Ciencias Médicas y Nutrición Salvador Zubirán, Ciudad de México, México
}

La disección endoscópica de la submucosa (DES) es una técnica mínimamente invasiva para el tratamiento de neoplasias tempranas del tracto gastrointestinal. La eficacia y seguridad de esta técnica han sido demostradas en diversos estudios, con tasas de R0 mayores al $80 \%$ y complicaciones $<10 \%$ (dependiendo del órgano) ${ }^{1}$. Es una técnica demandante en cuanto a habilidad endoscópica, a menudo consistiendo en procedimientos de duración prolongada. Es por ello que una proporción importante de los trabajos de investigación sobre DES se centran en aumentar la eficacia, disminuir la duración y mejorar la seguridad de esta técnica. A continuación se describen los trabajos más importantes sobre el tema, presentados en DDW 2021.

Kitagawa et al. compararon mediante un ensayo aleatorizado, el desempeño de 2 cuchillos, dual knife e IT knife nano, en términos de velocidad y seguridad del procedimiento durante la DES esofágica asistida por tracción (hilo y clip) ${ }^{2}$. Ellos incluyeron 50 pacientes con cáncer esofágico temprano a quienes aleatorizaron para DES con uno u otro cuchillo. El grupo de IT nano presentó de forma significativa menor duración del procedimiento (31 vs. 48 min; $p<0.01$ ) y duración de la disección de la submucosa (11 vs. $27 \mathrm{~min} ; \mathrm{p}<0.01$ ) que el grupo del dual knife. De forma adicional, se utilizó menos hemostasia por sangrado intraoperatorio en el grupo de IT knife vs. el grupo de dual knife (0 vs. $1 ; p<0.01$ ). Por otro lado, se observó exposición de la muscular propia en el $20 \%$ de los pacientes del grupo dual knife y en solo el $8 \%$ del grupo IT knife, aunque la diferencia no fue estadísticamente significativa. Es importante destacar que las tasas de resección en bloque, resección completa y eventos adversos fueron similares en ambos grupos. Este estudio nos demuestra que con maniobras sencillas como el cambiar de cuchillo durante DES, es factible aumentar la velocidad del procedimiento y disminuir eventos adversos. Será necesario realizar estudios adicionales con mayor número de pacientes, en otros segmentos del tubo digestivo, para poder tener datos más robustos.

El trabajo de Manabe, et al. tuvo por objetivo evaluar si el agregar un margen adicional de $1 \mathrm{~cm}$ durante la DES de los carcinomas de la unión esofagogástrica (CUEG) podría aumentar las tasas de márgenes laterales negativos durante la resección ${ }^{3}$. Lo anterior tomando en consideración que estas neoplasias pueden presentar una extensión tumoral subescamosa, difícil de identificar durante la evaluación endoscópica. Para ello, los autores llevaron a cabo un estudio prospectivo de factibilidad que incluyó pacientes con CUEG Siewert II, intramucosos, sin datos de compromiso ganglionar o metástasis a distancia. El margen de resección se estableció colocando marcas a $1 \mathrm{~cm}$ de la lesión. Se evaluaron 12 pacientes (5 con adenocarcinoma asociado a esófago de Barrett y 7 con adenocarcinoma de la unión). La tasa de resección completa fue del $91.7 \%$ (11/12). Todas las lesiones se identificaron por dentro de los márgenes laterales de $1 \mathrm{~cm}$, lo cual resultó en márgenes laterales negativos. La incidencia de extensión subescamosa del tumor fue de $75 \%$ (9/12), la mediana de la longitud de la extensión subescamosa fue de $5.7 \mathrm{~mm}$. La tasa de resección curativa fue del $66.7 \%(8 / 12)$ y 2 pacientes desarrollaron estenosis. Los autores concluyen que el adicionar un margen de $1 \mathrm{~cm}$ durante la DES para CUEG parece ser seguro y puede ayudar a alcanzar márgenes 
laterales negativos. Esta propuesta tendrá que ser validada por trabajos adicionales, sin embargo, su aplicación parece plausible, aumentando la probabilidad de tener márgenes laterales negativos en el tratamiento de una neoplasia que a menudo nos presenta dificultad para definir sus bordes en la evaluación endoscópica.

El grupo del Dr. Stavropoulos presentó los resultados de un estudio sobre la utilización de una plataforma de doble balón (DB) en DES de lesiones colónicas complejas $^{4}$. Este trabajo tuvo por objetivo evaluar los beneficios potenciales de la DES con DB en comparación con la DES convencional (DES-C) para el tratamiento de lesiones colónicas complejas. Ochenta lesiones tratadas con DES-DB fueron pareadas con 80 lesiones tratadas con DES-C para analizar duración del procedimiento y velocidad de la disección. Así mismo, se compararon 45 lesiones tratadas con DES-DB y sutura endoscópica del lecho de resección vs. 45 lesiones tratadas con DES-C y cierre del lecho con endoclips para determinar si la sutura endoscópica del lecho disminuye la duración de hospitalización (DH). No se encontraron diferencias en las tasas de eventos adversos graves. La velocidad de la disección fue mayor en el grupo de DES-DB (15.2 vs. $12.3 \mathrm{~cm}^{2} / \mathrm{h} ; \mathrm{p}=0.016$ ) y la duración total del procedimiento fue menor en el grupo DES-DB (58.6 vs. $76.3 \mathrm{~min} ; \mathrm{p}=0.039$ ). En cuanto a la comparación entre el uso de sutura endoscópica en el lecho de resección vs. uso de endoclips, la primera maniobra demostró disminuir la estancia hospitalaria (1.22 \pm 1.74 vs. $1.82 \pm 1.63$ días; $p=0.039$ ) y aumentar la proporción de pacientes egresados el mismo día del procedimiento (17 vs. 9 pacientes; $p=0.016$ ). La DES de lesiones colónicas representa un reto importante por la inestabilidad del endoscopio al realizar la resección, lo cual puede llevar a un aumento en la tasa de complicaciones y en la duración del procedimiento. Con la aplicación de esta plataforma de DB, la DES parece tornarse menos demandante, además de que permite utilizar la sutura endoscópica en colon, llevando a una disminución en la DH y egreso de los pacientes. Tendremos que esperar a que ambas plataformas (DB y sutura endoscópica) lleguen a nuestro país y a que se realicen más estudios que apoyen estos resultados.

Finalmente, el estudio de Kamigachi, et al. tuvo por objetivo evaluar los factores predictores de suspensión del procedimiento, resección en fragmentos y perforación durante la DES colorrectal en 11 instituciones japonesas con experiencia y habiendo completado la curva de aprendizaje del procedimiento ${ }^{5}$. Incluyeron
2,423 pacientes consecutivos con 2,592 tumores colorrectales; la tasa de resección en bloque fue del $96.4 \%$, con incidencia de suspensión, resección en fragmentos y perforación del $0.7,2.9$ y $3 \%$, respectivamente. En el análisis multivariante, los factores predictores de suspensión del procedimiento fueron: perforación durante la DES, invasión submucosa profunda (> $1,000 \mathrm{um}$ ), poca maniobrabilidad del endoscopio y fibrosis submucosa grave; los factores predictores de resección en fragmentos fueron: poca maniobrabilidad del endoscopio, fibrosis submucosa grave y duración prolongada del procedimiento ( $\geq 85 \mathrm{~min}$ ); y los factores predictores de perforación durante el procedimiento fueron: fibrosis submucosa grave, poca maniobrabilidad del endoscopio, duración prolongada del procedimiento ( $\geq 85 \mathrm{~min}$ ) y tamaño del tumor ( $\geq 40 \mathrm{~mm}$ ). Los autores concluyen que la fibrosis submucosa grave y la poca maniobrabilidad del endoscopio son los factores predictores que tienen en común la suspensión del procedimiento, la resección en fragmentos y la perforación durante el procedimiento, una vez estandarizada la técnica de la DES. Estos hallazgos nos llevan a reconsiderar a la DES colorrectal como un procedimiento que demanda alta habilidad endoscópica, que requiere de una curva de aprendizaje y que incluso una vez alcanzada esta curva, hay factores de riesgo que aumentarán la tasa de complicaciones 0 desenlaces no deseados durante el procedimiento.

\section{Financiamiento}

La presente investigación no ha recibido ayudas específicas provenientes de agencias del sector público, sector comercial o entidades sin ánimo de lucro.

\section{Conflicto de intereses}

Los autores declaran que no hay conflicto de intereses.

\section{Bibliografía}

\footnotetext{
1. Pimentel-Nunes $P$, Dinis-Ribeiro $M$, Ponchon $T$, Repici $A$, Vieth $M$, De Ceglie A, et al. Endoscopic submucosal dissection: European Society of Gastrointestinal Endoscopy (ESGE) Guideline. Endoscopy. 2015;47:829-54.

2. Kitagawa Y, Nankinzan R, Suzuki T. ID: 3521739 Randomized study of two endo-knives for the traction-assisted endoscopic submucosal dissection of esophageal cancer. Gastrointest Endosc [Internet]. 2021;93(6 Suppl):AB293-AB294. Disponible en: https://www.giejournal.org/article/ S0016-5107(21)01241-4/abstract

3. Manabe T, Nagami Y, Yamamoto K, Hirano S, Kono M, Ociai T, et al. ID: 3517856 An extra 1-cm safety margin during endoscopic submucosal dissection can control subsquamous tumor extension of adenocarcinoma in the esophagogastric junction: a prospective feasibility study. Gastrointest Endosc [Internet]. 2021;93(6 Suppl):AB280. Disponible en: https:// www.giejournal.org/article/S0016-5107(21)00830-0/abstract
} 
4. Stavropoulos SN, Parsa N, Widmer JL, Badshah MB, Alansari TH, Khodorskiy DO, et al. ID: 3526950 Use of a double balloon platform facilitates endoscopic submucosal dissection (esd) of complex colon lesions and decreases post esd length of stay(los): a single center case matched study. Gastrointest Endosc [Internet]. 2021;93(6 Suppl):AB93. Disponible en: https://www. giejournal.org/article/S0016-5107(21)00482-X/abstract
5. Kamigaichi $Y$, Oka S, Nagata S, Kunihiro M, Kuwai T, Hiraga $Y$, et al. ID: 3520676 Predictive factors for interruption, piecemeal resection, and perforation after standardization of colorrectal endoscopic submucosal dissection. Gastrointest Endosc [Internet]. 2021:93(6 Suppl):AB94. Disponible en: https://www.giejournal.org/article/S0016-5107(21)00484-3/ abstract 